\title{
Subacute Intracranial Subdural Hematoma 40 Days After Spinal Anesthesia: A Case Report
}

\author{
Hamid Reza Hatamabadi, ${ }^{1,2}$ Ali Tabatabaey, ${ }^{3}$ and Behnaz Tajvidi ${ }^{2,}$ \\ ${ }^{1}$ Safety Promotion and Injury Prevention Research Center, Shahid Beheshti University of Medical Sciences, Tehran, IR Iran \\ ${ }^{2}$ Department of Emergency, Imam Hossein Hospital, Shahid Beheshti University of Medical Sciences, Tehran, IR Iran \\ ${ }^{3}$ Department of Emergency Medicine, Qom University of Medical Sciences and Health Services, Qom, IR Iran \\ "Corresponding author: Behnaz Tajvidi, Department of Emergency, Imam Hossein Hospital, Shahid Beheshti University of Medical Sciences, Tehran, IR Iran. Tel: \\ +98-2173432380, Fax: +98-2177557069, E-mail: hhatamabadi@yahoo.com
}

Received 2014 November 16; Revised 2015 January 20; Accepted 2015 July 14.

\begin{abstract}
Introduction: Subdural hematoma is a serious but rare complication of spinal anesthesia. Differentiation between post-dural puncture headache (PDPH) and subdural hematoma can be difficult.

Case Presentation: A 32-year-old female patient underwent elective cesarean section under spinal anesthesia. Ten days postoperatively, the patient developed symptoms of headache and nausea, which progressed over time. The patient was brought to the emergency department on the 40th postoperative day with hemiparesis on the left side of the body. Brain MRI revealed a sub acute subdural hematoma. Progressive neurologic signs and evidence of mass effect and cerebral shifting on brain imaging were clear indications for surgical intervention. The patient recovered completely after surgical decompression.

Conclusions: A high index of suspicion for the pattern and characteristics of headache, and a meticulous neurologic examination, can help the emergency physician recognize the serious entity of subdural hematoma.
\end{abstract}

Keywords: Anesthesia, Spinal, Complications, Hematoma, Subdural, Post-Dural Puncture Headache

\section{Introduction}

In spinal anesthesia, analgesics are introduced into the subarachnoid spinal space using sterile technique. The procedure is considered a safe and effective alternative to general anesthesia when the surgical site is located in the lower extremities, perineum, or lower abdominal wall. Post-dural puncture headache(PDPH) is a well-known complication of spinal anesthesia that is usually treated with rest, hydration, and simple analgesics (1). Though in itself a benign entity, PDPH might be a sign of a rarer but more serious complication, intracranial hemorrhage $(2,3)$. Patients with complaints of PDPH often seek treatment at the emergency department (ED). When PDPH persists or is accompanied by other neurologic findings, the emergency physician must investigate for an intracranial hemorrhage. Here, we report the case of a sub acute intracranial subdural hematoma (SDH) diagnosed 40 days after spinal anesthesia.

\section{Case Presentation}

A 32-year-old woman was brought to our ED complaining of headache and numbness in her upper left arm and face. She had undergone an elective cesarean section with spinal analgesia at another hospital 40 days prior to presentation to our ED. The headache was defined as diffuse with vague characteristics. The patient's pregnancy had been normal and well-controlled. She had no history of hypertension, migraine, malignancy, blood disease, infection, or neurological disease. She had no history of head trauma, primary headache, or coagulation abnormalities. There was no familial history of coagulation disorders or cerebrovascular hemorrhage.

The spinal anesthesia had been conducted by puncturing the L4 - L5 space with a 26-gauge spinal needle and injecting $10 \mathrm{mg}$ of $0.5 \%$ bupivacaine. The puncture was successful on the first attempt, and the course of anesthesia was without incident. Her intraoperative vital signs were normal and the surgery was completed uneventfully. After surgery, no restrictions on position were implemented and the patient was placed in a position of comfort. Full recovery was achieved and she was discharged on the second day after the operation. She had no headache at the time of discharge. The patient followed a relative bed rest regimen at home and refrained from heavy labor. She did not return to work.

Seven to ten days later, the patient developed a mild diffuse headache that did not subside with analgesia and bed rest. The severity of the headache gradually increased.

Copyright @ 2015, Tehran University of Medical Sciences. This is an open-access article distributed under the terms of the Creative Commons Attribution-NonCommercial 4.0 International License (http://creativecommons.org/licenses/by-nc/4.0/) which permits copy and redistribute the material just in noncommercial usages, provided the original work is properly cited. 
Over the following few weeks, numbness developed in the left upper limb and face, at which time the patient was brought to the ED. On presentation forty days postoperatively, she complained of an intense generalized headache that worsened upon sitting upright and in the evenings. On examination, she was conscious and oriented. There was no sign of fever or meningeal irritation. Fundoscopic examination was normal. She had no neck rigidity or Kerning's sign. The motor force of all limbs was 5.5, but the left plantar reflex was increased. Her BP was $120.80 \mathrm{mmHg}$, and no fever was detected.

Laboratory data revealed normal hemoglobin, white blood cell count, platelet count, PT, PTT, and INR. CT scan of the head was performed, which displayed an isodense to hypodense crescent-shaped space-occupying lesion in the right parietal lobe close to the vertex (Figure1). The diagnosis was confirmed with an MRI of the head, which showed hyperintensity in the subdural region over the right parietal lobe on both the T1- and T2-weighted images, consistent with a late sub acute hemorrhage. Mild mass effect on the ipsilateral ventricle and the midline was also evident (Figures 2 and 3).

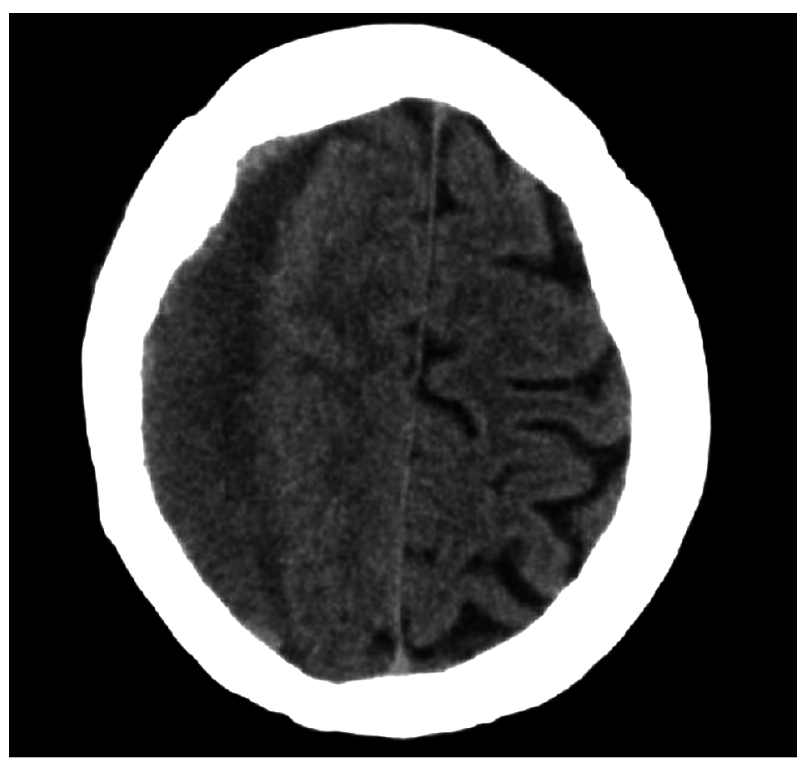

Figure 1. CT Scan of Head Revealing Isodense to Hypodense Mass on the Right Side

After emergent consultation with a neurosurgeon, the patient was admitted to the neurosurgery ward, and clot evacuation was achieved using burr-hole placement. She was discharged home three days later, and had an uneventful recovery with full resolution of her symptoms. Followup MRI and CT scans were normal, and she remains fit and well.

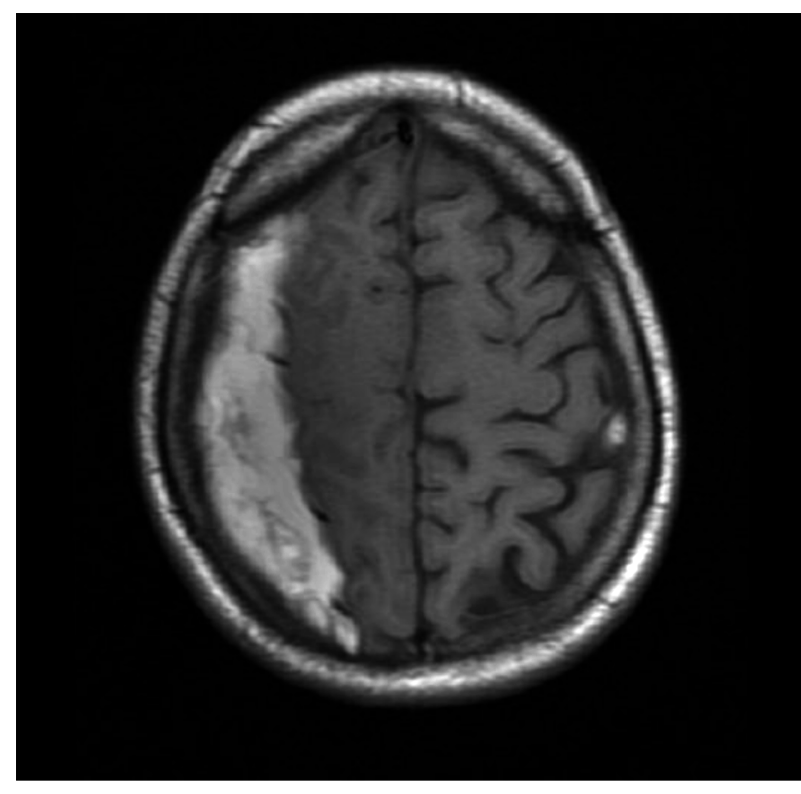

Figure 2. T1 Axial MRI Image Revealing Hyperintensity on The Right Side

Figure 3. Coronal T2 Image Showing Hyperintensity Near the Vertex on The Right Side

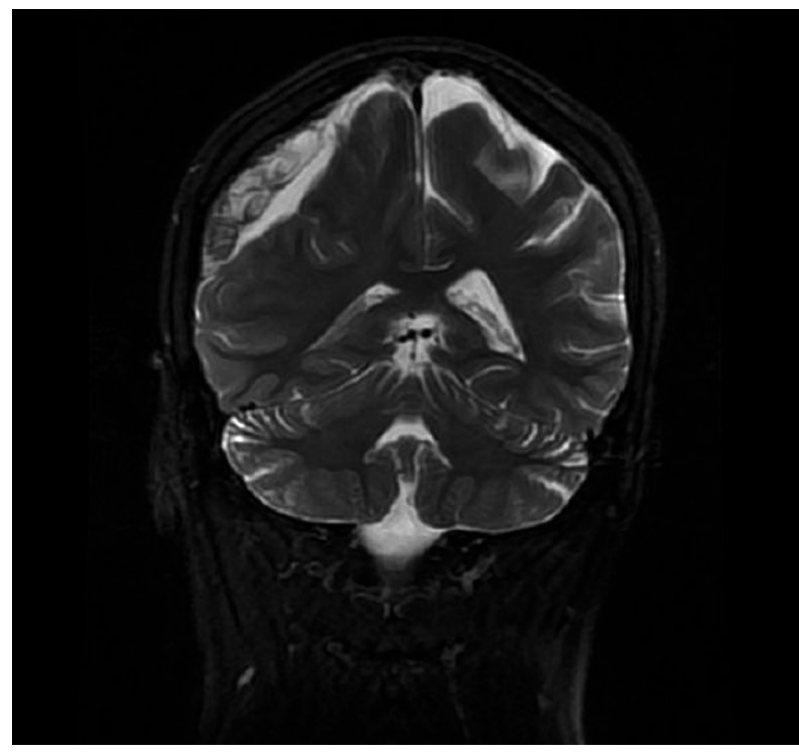

Note the near-collapse of the right ventricle due to mass effect.

\section{Discussion}

Spinal anesthesia is an accepted and often favored method to achieve anesthesia in obstetric patients $(4,5)$. However, women have been found to be more susceptible to PDPH, a frequent and benign side effect of dural punc- 
ture (6). The mechanism for PDPH is not fully understood, but continued cerebrospinal fluid (CSF) leakage, leading to a reduction in CSF pressure, is often blamed $(1,6,7)$. The international headache society has developed a list of criteria to help differentiate PDPH from other, more serious, complications of dural puncture (8). According to these criteria, in PDPH, the pain worsens or develops within 15 minutes of assuming an upright position, and improves within a similar period after the individual lays down. It develops within 5 days after the puncture and disappears spontaneously within one week, or up to 48 hours after an epidural blood patch is employed. In the case presented here, the initial diagnosis had been PDPH. However, by the time the patient arrived at our ED, more than a month had passed since the puncture, and traditional remedies had failed to improve her headache, so further investigation was justified. In a study of 25 patients by Zeidan et al. the time between lumbar puncture and presentation ranged from six hours to 29 weeks. Nine cases were recognized 25 - 50 days after the suspected procedure (7).

An incidence of 1 in 500,000 obstetric procedures has been estimated for intracranial SDH following spinal anesthesia (9), but most authors believe that the true incidence of SDH after dural puncture is unknown and that many cases go unreported. The phenomenon has been reported to involve acute, acute-on-chronic, sub acute, and chronic forms (7, 10-12). In the Zeidan case study, SDH after spinal anesthesia occurred most frequently on the left side of the brain (13 cases), followed by six right-sided, four bilateral, and two intracerebral cases (7). In our case, the bleeding was right-sided and presented in the late subacute phase.

In reviewing 35 cases of intracranial hemorrhage following spinal anesthesia, Amorim et al. found that 15 of these patients did not have any recognized predisposing factors. Among those who did have such risk factors, the most common were pregnancy, multiple punctures, use of anticoagulants, intracranial vascular abnormalities, and brain atrophy (13). The only attributable risk factor in our patient was pregnancy; a single puncture had been used and she had no signs of a hemostatic defect.

Differentiation between the neurological symptoms of intracranial hypotension (PDPH) and SDH can be difficult. In the cases presented by Amorim, in addition to headache as the chief complaint, $89 \%$ of the patients presented with at least one of the following signs: vomiting, diplopia, cognitive changes or altered mental status, or focal neurologic signs (13). The authors suggested that the presence of any of these findings should prompt the physician to search for causes other than PDPH. Also, a change in the headache characteristics from postural to non-postural has been mentioned as a warning sign that intracranial hemorrhage may be complicating simple intracranial hy- potension (7). In our patient, the pain was not relieved in the supine position, but more importantly, the presence of focal neurologic deficits, in the form of a positive Babinski sign and hemiparesis, pointed toward a more serious diagnosis.

As with any other SDH, the management is either conservative or surgical. Small hematomas often resolve spontaneously, and therefore the risk of surgical evacuation is not justified. It is believed that early blood patching after the presentation of PDPH may decrease the risk of subdural bleeding through prevention of CSF loss (14). On the other hand, introduction of an epidural blood patch in the presence of intracranial hemorrhage may lead to rebound intracranial hypertension and deterioration (15). Nonetheless, Davies et al. reported treating a case of recognized SDH following dural puncture with an epidural blood patch (16). In cases of larger hematomas, surgical evacuation of the clot is indicated. In the Amorim study, 27 of the 35 cases required surgical drainage (13), and in the Zeidan series, surgery was performed in 20 of 25 patients (7). In the patient we present here, progressive neurologic signs and evidence of mass effect and cerebral shifting on brain imaging were clear indications for surgical intervention.

Spinal anesthesia-related SDH is a potentially lifethreatening complication of this well-accepted procedure. It can develop with seemingly innocent signs that are usually attributed to PDPH. A high index of suspicion and close attention to the pattern and characteristics of the headache, coupled with a meticulous neurologic exam, can help the emergency physician recognize this serious entity.

\section{Acknowledgments}

We would like to thank the neurosurgery faculty and residents of Shahid Beheshti University of Medical Sciences at Emam Hossein hospital for their participation and useful hints, which made this research a fruitful experience.

\section{Footnote}

Authors' Contribution: Study concept and design: Hamid Reza Hatamabadi; drafting of the manuscript: Behnaz Tajvidi; critical revision of the manuscript for important intellectual content: Behnaz Tajvidi; administrative, technical, and material support: Ali Tabatabaey; study supervision: Hamid Reza Hatamabadi. 


\section{References}

1. Wrobel M, Volk T. Post-dural puncture headache. Anesth Pain Med. 2012;1(4):273-4. doi: 10.5812/aapm.3610. [PubMed: 24904817].

2. Schweiger V, Zanconato G, Lonati G, Baggio S, Gottin L, Polati E. Intracranial subdural hematoma after spinal anesthesia for cesarean section. Case Rep Obstet Gynecol. 2013;2013:253408. doi: 10.1155/2013/253408. [PubMed: 24455348].

3. Machurot PY, Vergnion M, Fraipont V, Bonhomme V, Damas F. Intracranial subdural hematoma following spinal anesthesia: case report and review of the literature. Acta Anaesthesiol Belg. 2010;61(2):636. [PubMed: 21155439].

4. Azmude A, Agha'amou S, Yousefshahi F, Berjis K, Mirmohammad'khani M, Sadaat'ahmadi F, et al. Pregnancy outcome using general anesthesia versus spinal anesthesia for in vitro fertilization. Anesth Pain Med. 2013;3(2):239-42. doi: 10.5812/aapm.11223. [PubMed: 24282775].

5. Lak M, Forouzanmehr M, Doudangeh F. Spinal versus epidural anesthesia complications, outcomes and pain relieving capacity. Trauma Mon. 2008;13(2):153-61.

6. Valenca MM, Amorim JA, Moura TP. Why don't all individuals who undergo dura mater/arachnoid puncture develop postdural puncture headache?. Anesth Pain Med. 2012;1(3):207-9. doi: 10.5812/kowsar.22287523.3616. [PubMed: 24904799].

7. Zeidan A, Farhat O, Maaliki H, Baraka A. Does postdural puncture headache left untreated lead to subdural hematoma? Case report and review of the literature. Int J Obstet Anesth. 2006;15(1):50-8. doi: 10.1016/j.ijoa.2005.07.001. [PubMed:16256333].

8. Headache Classification Subcommittee of the International Headache Society. . The International Classification of Headache
Disorders. Cephalalgia. 2004;24 Suppl 1:9-160. [PubMed: 14979299].

9. Scott DB, Hibbard BM. Serious non-fatal complications associated with extradural block in obstetric practice. $\mathrm{Br} J$ Anaesth 1990;64(5):537-41. [PubMed: 2354090].

10. Hagino T, Ochiai S, Watanabe Y, Senga S, Saito M, Naganuma H, et al. Acute onset of intracranial subdural hemorrhage five days after spinal anesthesia for knee arthroscopic surgery: a case report. J Med Case Rep. 2012;6:75. doi: 10.1186/1752-1947-6-75. [PubMed: 22394604].

11. Tan ST, Hung CT. Acute-on-chronic subdural haematoma: a rare complication after spinal anaesthesia. Hong Kong Med J. 2003;9(5):384-6. [PubMed: 14530536].

12. Martínez-Lage JF, Verdú MT, Alonso B, Sánchez-Ortega JL, GarciaCandel A. Non-surgical management of intracranial subdural hematoma complicating spinal anesthesia. Neurocirugía. 2007;18(1):40-3. doi: 10.1016/s1130-1473(07)70309-3. [PubMed: 17393045].

13. Amorim JA, Remigio DS, Damazio Filho O, de Barros MA, Carvalho VN, Valenca MM. Intracranial subdural hematoma post-spinal anesthesia: report of two cases and review of 33 cases in the literature. Rev Bras Anestesiol. 2010;60(6):620-9. doi: 10.1016/S0034-7094(10)700775. [PubMed: 21146058].

14. Reynolds AF, Slavin L. Postpartum acute subdural hematoma; a probable complication of saddle block analgesia. Neurosurgery. 1980;7(4):398-9. [PubMed: 7442983].

15. Nolte $\mathrm{CH}$, Lehmann TN. Postpartum headache resulting from bilateral chronic subdural hematoma after dural puncture. Am J Emerg Med. 2004;22(3):241-2. [PubMed: 15138975].

16. Davies JM, Murphy A, Smith M, O'Sullivan G. Subdural haematoma after dural puncture headache treated by epidural blood patch. $\mathrm{Br} J$ Anaesth. 2001;86(5):720-3. [PubMed: 11575351]. 